\title{
Análise da Qualidade dos Artigos Científicos da Área de Marketing no Brasil: As Pesquisas Survey na Década de 90
}

\author{
Lurdes Marlene Seide Froemming \\ Fernando Bins Luce \\ Marcelo Gattermann Perin \\ Cláudio Hoffmann Sampaio \\ Sedinei José Nardelli Beber \\ Guilherme Trez
}

\section{RESUMO}

Este artigo apresenta um levantamento das pesquisas empíricas do tipo survey publicadas na década de 90 nos periódicos Revista de Administração de Empresas da Fundação Getúlio Vargas (RAE) e Revista de Administração da Universidade de São Paulo (RAUSP), além dos anais dos Encontros Anuais da Associação Nacional dos Programas de Pós-Graduação em Administração (ENANPAD). Entre as variáveis analisadas para cada artigo estão: natureza da pesquisa, embasamento conceitual e questão de pesquisa, desenho de pesquisa, instrumento de medida e coleta de dados, confiabilidade e validade dos constructos, tipos de análises dos dados empregados e apresentação dos resultados. A partir desta análise e do cruzamento entre as variáveis, algumas conclusões sobre a qualidade do conjunto de artigos avaliados são emitidas.

Palavras-chaves: marketing; pesquisa tipo survey; metodologia de pesquisa científica.

\begin{abstract}
This paper presents an inventory of survey research published in the years 90 . This production is available in the periodics Revista de Administração de Empresas da Fundação Getúlio Vargas (RAE) and Revista de Administração da Universidade de São Paulo (RAUSP) and in the anuals of Encontros Anuais da Associação Nacional dos Programas de Pós-Graduação em Administração (ENANPAD). The analised variables for each paper were: nature of research, theorical base and research problem, research design, measuring instruments, reliability and validity from the constructs, kind of data analysis and finding presentations. Considering this analysis and correlations of the variables, some conclusions are emitted about the quality of the set of evaluated papers.
\end{abstract}

Key words: marketing; survey research; scientific research methodology. 


\section{INTRODUÇÃO}

Nos últimos dois anos, alguns artigos publicados nos anais dos Encontros Anuais da Associação Nacional dos Programas de Pós-Graduação em Administração (ENANPAD) procuraram trazer à tona a discussão sobre a pesquisa e produção científica da comunidade acadêmica brasileira, especificamente na área de administração. Além dos artigos, alguns painéis e mesas redondas ${ }^{(1)}$ vêm analisando desde qualidade até a continuidade dos trabalhos realizados e publicados em âmbito nacional dentro da ciência da administração.

Mais recentemente, em um primeiro documento publicado no número anterior da Revista de Administração Contemporânea (RAC), apresentamos um inventário dos artigos científicos de marketing publicados nos periódicos Revista de Administração de Empresas da Fundação Getúlio Vargas (RAE) e Revista de Administração da Universidade de São Paulo (RAUSP), além dos anais do ENANPAD, durante a década de 90 . O referido artigo faz parte de um conjunto de estudos que visa a analisar a qualidade das publicações científicas brasileiras na área de marketing.

Nesta linha, este segundo trabalho apresenta um levantamento das pesquisas empíricas do tipo survey publicadas na década de 90 nos anais do ENANPAD, dentro da área de marketing. Para tanto, analisam-se as estratégias e as metodologias utilizadas nestas publicações. O método aplicado para a pesquisa é descrito no item 2. No item 3 são relatados os resultados do estudo, bem como identificados e estabelecidos cruzamentos entre as variáveis analisadas. Por fim, o item 4 apresenta as principais conclusões oriundas dos resultados obtidos com a pesquisa, com alguns comentários sobre a qualidade dos artigos publicados.

\section{Método}

O procedimento metodológico utilizado foi o de desk research, envolvendo o conjunto de artigos científicos da área de marketing que utilizaram pesquisa empírica tipo survey, publicados nos anais dos dez últimos ENANPAD, na RAUSP e na RAE, durante a década de 90. Essas revistas e anais de congresso foram escolhidos para a análise por terem sido considerados os veículos brasileiros mais representativos daquilo que se produz e publica em termos científicos dentro da área de marketing. 
O processo de identificação e seleção de artigos resultou em uma relação de 124 artigos de pesquisa survey para a análise. A Tabela 1 apresenta a freqüência de artigos analisados por ano e publicação. Nota-se que o ENANPAD, dentre os periódicos analisados, concentra a maioria dos artigos analisados neste tipo de pesquisa $(87,09 \%)$.

Tabela 1: Freqüência de Artigos por Periódico e Ano de Publicação

\begin{tabular}{c|ccc|c}
\hline Ano & \multicolumn{3}{|c|}{ Survey } & Total \\
\hline & ENANPAD & RAE & RAUSP & \\
$\mathbf{9 0}$ & 6 & 0 & 1 & $\mathbf{7}$ \\
$\mathbf{9 1}$ & 7 & 0 & 1 & $\mathbf{8}$ \\
$\mathbf{9 2}$ & 6 & 0 & 0 & $\mathbf{6}$ \\
$\mathbf{9 3}$ & 9 & 0 & 3 & $\mathbf{1 2}$ \\
$\mathbf{9 4}$ & 10 & 0 & 1 & $\mathbf{1 1}$ \\
$\mathbf{9 5}$ & 7 & 1 & 0 & $\mathbf{8}$ \\
$\mathbf{9 6}$ & 11 & 1 & 1 & $\mathbf{1 3}$ \\
$\mathbf{9 7}$ & 15 & 3 & 0 & $\mathbf{1 8}$ \\
$\mathbf{9 8}$ & 19 & 1 & 1 & $\mathbf{2 1}$ \\
$\mathbf{9 9}$ & 18 & 2 & 0 & $\mathbf{2 0}$ \\
\hline Total & 108 & 8 & 8 & $\mathbf{1 2 4}$ \\
\hline
\end{tabular}

O processo de análise dos 124 artigos de pesquisas survey constituiu-se da verificação dos elementos metodológicos básicos de investigações empíricas, que refletem a qualidade científica de uma pesquisa. As variáveis avaliadas para cada artigo selecionado, adaptadas de Hoppen et al. (1997), foram as seguintes:

. natureza da pesquisa;

- embasamento conceitual e questão de pesquisa, analisando-se: justificativa, teorias de base, objetivo/questão de pesquisa e hipóteses/pressupostos de base;

. desenho de pesquisa, avaliando-se: modelo de pesquisa, tipo de estudo, mix de métodos, operacionalização das variáveis e descrição dos procedimentos metodológicos;

instrumento de medida e coleta de dados, analisando-se: etapa exploratória, validade de conteúdo, etapa quantitativa, tipo de dados, tipo de amostra, taxa de resposta, teste do viés de não respondentes e caracterização da amostra;

. confiabilidade e validade dos constructos, avaliando-se: confiabilidade, validade convergente e validade discriminante; 
. tipos de análise dos dados empregados; e

- apresentação dos resultados, analisando-se: validade externa, validade nomológica, limites do estudo, recomendações para futuras pesquisas e recomendações aplicadas.

Os artigos foram analisados por um grupo de seis pesquisadores de marketing, sendo que cada artigo foi examinado por apenas um avaliador; contudo, buscando-se maior validade para o estudo, os critérios de análise foram amplamente discutidos entre os membros da equipe de avaliadores e sintetizados no formulário anexo (vide Anexo 1), que foi utilizado para cada um dos artigos. Além disto, todas as avaliações foram discutidas entre os pesquisadores em reuniões do grupo. Os dados, uma vez coletados e discutidos, foram tabulados e processados pelos softwares SPSS, Le Sphinx Plus e Excel.

\section{Resultados das ANÁlises}

A seguir são descritos os principais resultados relativos às variáveis analisadas, considerando os 124 artigos com pesquisa survey publicados no Brasil na área de marketing durante a década de 90 .

\section{Natureza da Pesquisa}

A natureza da pesquisa foi classificada em exploratória, descritiva ou causal, conforme Churchill Jr. (1999).

No caso de uma pesquisa exploratória, trata-se de aprofundar conceitos preliminares, muitas vezes inéditos, buscando a geração de idéias ou insights. Seu objetivo básico é desenvolver hipóteses e proposições que irão redundar em pesquisas complementares. Por outro lado, uma pesquisa descritiva tem como objetivo mapear a distribuição de um fenômeno na população estudada. Em geral, busca determinar a freqüência com que algo ocorre ou a relação entre duas variáveis, sendo tipicamente guiada por uma hipótese inicial. A natureza causal, por sua vez, objetiva o teste de uma teoria e suas relações de causa e efeito.

A Tabela 2 descreve a natureza das pesquisas em marketing publicadas nos periódicos analisados. Destaca-se, quanto a esta variável, o elevado percentual da natureza descritiva das pesquisas survey realizadas na área de marketing, representando aproximadamente $61 \%$. 
Tabela 2: Freqüência de Artigos por Natureza de Pesquisa

\begin{tabular}{lcc}
\hline Natureza & N & Perc. \\
\hline Exploratória & 37 & $29,84 \%$ \\
Descritiva & 76 & $61,29 \%$ \\
Causal & 11 & $8,87 \%$ \\
\hline Total & $\mathbf{1 2 4}$ & \\
\hline
\end{tabular}

\section{Apresentação e Relevância do Problema de Pesquisa}

Esta categoria trata de dimensões que identificam a fundamentação conceitual utilizada e a relevância do estudo para a área, bem como os objetivos da pesquisa apresentada e suas hipóteses. A Tabela 3 apresenta os resultados da análise quanto a este item.

Tabela 3: Freqüência de Artigos por Apresentação e Relevância do Problema de Pesquisa

\begin{tabular}{llcc}
\hline \multirow{2}{*}{ Justificativa } & & N & Perc. \\
\hline \multirow{2}{*}{ Teorias de Base } & Sim & 113 & $91,13 \%$ \\
\cline { 2 - 4 } & Aão & 11 & $8,87 \%$ \\
\cline { 2 - 4 } & Superficial & 10 & $8,06 \%$ \\
\cline { 2 - 4 } Objetivo e Questão de Pesquisa & Aprofundada & 50 & $51,61 \%$ \\
\hline & Sim & 123 & $99,32 \%$ \\
\hline Hipóteses e Pressupostos de Base & São & 1 & $0,81 \%$ \\
\cline { 2 - 4 } & Sim & 46 & $37,10 \%$ \\
\hline & Não & 78 & $62,90 \%$ \\
\hline
\end{tabular}

Em relação à justificativa, registrou-se apenas se o artigo analisado apresentava ou não justificativa ou relato de importância da pesquisa. A teoria de base foi considerada aprofundada, quando o artigo continha uma descrição detalhada do fenômeno em estudo do ponto de vista dos principais autores da área ou de uma linha de estudo específica de um grupo de autores. Caso a descrição do fenômeno apresentasse pouco detalhamento, mas ainda dentro do fenômeno em estudo, considerou-se a teoria de base como superficial. Em qualquer outro caso, considerou-se a mesma como ausente ou não relacionada. 
Quanto ao objetivo e à questão de pesquisa, avaliou-se se o artigo descrevia ou não o problema de pesquisa e/ou objetivo geral. O problema de pesquisa implica uma questão que envolve uma dificuldade teórica ou prática, para a qual se quer encontrar uma resposta ou solução. Da mesma forma, as hipóteses ou pressupostos de base foram apenas considerados como existentes (declarados) ou não para cada artigo analisado.

Conforme a Tabela 3, os artigos, em termos gerais, demonstraram preocupação com a apresentação e a relevância do problema de pesquisa. Grande parte dos artigos apresentou justificativa do estudo (91,13\%), bem como objetivos e questão de pesquisa $(99,19 \%)$.

A maioria dos artigos $(91,94 \%)$ apresentou a fundamentação teórica para a pesquisa descrita, sendo que em $40,32 \%$ deles a teoria de base foi considerada como aprofundada e em 51,61\% como superficial.

Por outro lado, destaca-se que apenas 46 artigos $(37,10 \%)$ apresentaram hipóteses ou pressupostos de base. Este número pode ser considerado baixo, se contrastado com o percentual de pesquisas de natureza descritiva e causal $(70,16 \%$ dos artigos), as quais sugerem a existência de hipóteses ou pressupostos de base (Sampieri, Collado e Lucio, 1998; Churchill Jr., 1999).

\section{Desenho de Pesquisa}

Esta categoria engloba as dimensões relacionadas com o modelo de pesquisa, tipo de estudo, utilização de mix de métodos, operacionalização das variáveis do estudo e descrição dos procedimentos metodológicos utilizados. A presença destas dimensões foi verificada em cada um dos artigos analisados. A Tabela 4 resume os resultados obtidos.

Tabela 4: Freqüência de Artigos por Desenho de Pesquisa

\begin{tabular}{|c|c|c|c|}
\hline & & $\mathbf{N}$ & Perc. \\
\hline \multirow[t]{2}{*}{ M odelo de Pesquisa } & $\mathrm{S}$ im & 20 & $16,13 \%$ \\
\hline & $\mathrm{N}$ ão & 104 & $83,87 \%$ \\
\hline \multirow[t]{2}{*}{$T$ ipo de E studo } & Long itud inal & 4 & $3,23 \%$ \\
\hline & C orte Transversal & 120 & $96,77 \%$ \\
\hline \multirow[t]{2}{*}{$M$ ix de $M$ étodos } & $\mathrm{S} \mathrm{im}$ & 1 & $0,81 \%$ \\
\hline & $\mathrm{N}$ ão & 123 & $99,19 \%$ \\
\hline \multirow[t]{2}{*}{ O pe racionaliza ção das $\mathrm{V}$ ariáve is } & $\mathrm{S} \mathrm{im}$ & 47 & $37,90 \%$ \\
\hline & $\mathrm{N}$ ão & 77 & $62,10 \%$ \\
\hline \multirow[t]{2}{*}{ D escrição dos P roc. M etodológicos } & $\mathrm{S} \mathrm{im}$ & 112 & $90,32 \%$ \\
\hline & $\mathrm{N}$ ão & 12 & $9,68 \%$ \\
\hline
\end{tabular}


Como modelo de pesquisa considerou-se o posicionamento do objeto em estudo dentro da base teórica ou de uma descrição mais ampla do fenômeno estudado, podendo ser gráfico ou não.

Em relação ao tipo de estudo, avaliou-se se os dados foram obtidos em momentos distintos no tempo (estudo longitudinal) ou em apenas um momento (corte transversal).

Quanto ao mix de métodos levou-se em conta a utilização de mais de um método de pesquisa, para avaliar a mesma questão de pesquisa. Note-se que este mix de métodos não se refere à diversidade dos métodos de coleta de dados.

A operacionalização das variáveis referiu-se à descrição das variáveis consideradas no fenômeno estudado, além da sua forma de mensuração.

Quanto à descrição dos procedimentos metodológicos utilizados, registrou-se apenas se o artigo descrevia ou não esses procedimentos.

A análise da Tabela 4 revela que, de modo geral, nos artigos analisados, há pouca ênfase no refinamento do desenho de pesquisa. Verifica-se que somente $16,13 \%$ dos artigos apresentaram o modelo de pesquisa, posicionando, graficamente ou não, o objeto em estudo dentro da base teórica ou de uma descrição mais ampla do fenômeno estudado. Em relação ao tipo de estudo, observa-se que quase a totalidade dos artigos analisados $(96,77 \%)$ utilizaram estudos do tipo corte transversal, isto é, não mediram as variáveis em momentos distintos no tempo. A utilização de uma abordagem multimétodo para avaliar a mesma questão de pesquisa praticamente não foi utilizada, uma vez que essa abordagem apareceu em apenas 1 dos 124 artigos analisados. Por fim, a maior parte dos artigos $(62,10 \%)$ não apresentaram a operacionalização das variáveis do estudo, isto é, não descreveram essas variáveis nem indicaram a forma de mensuração delas.

Já em relação à descrição dos procedimentos metodológicos, nota-se uma preocupação dos pesquisadores em descrever as etapas metodológicas desenvolvidas no estudo $(90,32 \%)$.

\section{Instrumentos de Medida e Coleta de Dados}

Esta categoria trata de dimensões que identificam a existência de uma etapa exploratória para a elaboração do instrumento de coleta de dados, da validade de conteúdo, do tipo de instrumento utilizado para a coleta de dados, do tipo de dado coletado, da existência e do tipo de amostragem, da verificação de viés dos não respondentes e da caracterização da amostra. As Tabelas 5 a 12 descrevem os resultados dessa categoria. 
Quanto à etapa exploratória, levou-se em consideração se o artigo apresentava ou não esta etapa, verificando-se, ainda, a estratégia qualitativa utilizada para a elaboração do instrumento de coleta de dados.

Em relação à validade de conteúdo/face, que verifica se o conteúdo de uma escala representa aquilo que se procura mensurar (Malhotra, 1996), abordando a clareza e a adequação dos enunciados e indicadores utilizados no instrumento de coleta de dados para a mensuração das variáveis do estudo (Hair Jr. et al., 1998), considerou-se se o artigo apresentava ou não a validade de conteúdo, bem como qual método foi utilizado para estabelecer a validade.

O modo de coleta de dados referiu-se ao tipo ou tipos de estratégia utilizados para a coleta de dados na pesquisa survey.

No item tipos de dados, avaliou-se se os dados foram coletados especificamente para o estudo (dados primários) ou se já estavam anteriormente disponibilizados (dados secundários). Levou-se em consideração, também, a possibilidade de utilização de ambos os tipos num mesmo estudo.

Em relação à amostra e ao processo de amostragem, considerou-se se o artigo apresentava ou não um processo de amostragem, e se esse processo era ou não probabilístico. Em amostras probabilísticas, cada elemento da população tem uma chance conhecida e diferente de zero de ser incluído na amostra (Churchill Jr., 1999). Contrariamente, em amostras não probabilísticas, não há maneira de ser estimada a probabilidade de um elemento da população ser incluído na amostra e de se certificar que a amostra é representativa da população (Churchill Jr., 1999). Verificou-se, ainda, se a amostra tinha sido caracterizada, com ou sem comparação com a população do estudo.

Por fim, levou-se em consideração a aplicabilidade e a utilização do teste do viés para os não respondentes, para verificar a existência ou não de falha em obter informações de alguns elementos da população que não foram selecionados e designados para a amostra, verificando se aqueles que não responderam ao instrumento de coleta de dados utilizado na pesquisa são significativamente diferentes daqueles que responderam (Churchill Jr., 1999).

A análise das Tabelas 5 e 6 indica um número elevado de ausências na descrição dos procedimentos utilizados para a geração do instrumento de coleta das pesquisas survey $(34,68 \%)$, bem como na validade de conteúdo/face deles $(54,03 \%)$. Por outro lado, nota-se um considerável número de pesquisas que utilizaram instrumentos gerados em outras pesquisas $(25,00 \%)$, demonstrando uma possível seqüência de trabalhos e/ou validações de pesquisas externas no ambiente brasileiro. 
Tabela 5: Tipo de Instrumento, Estratégia de Aplicação e Modo de Coleta - Etapa Exploratória

\begin{tabular}{lcc}
\hline Instrumento & $\mathbf{N}^{(2)}$ & Perc. \\
\hline Ausente & 43 & $34,68 \%$ \\
Instrumento gerado em outra pesquisa & 31 & $25,00 \%$ \\
Entrevista em profundidade & 30 & $24,19 \%$ \\
Focus group & 8 & $6,45 \%$ \\
Outros & 19 & $15,32 \%$ \\
\hline
\end{tabular}

Tabela 6: Validade do Conteúdo/Face

\begin{tabular}{lcc}
\hline Ins trumento & $\mathbf{N}^{(2)}$ & Perc. \\
\hline Ausente & 67 & $54,03 \%$ \\
Pré-teste & 38 & $30,65 \%$ \\
Especialistas & 14 & $11,29 \%$ \\
Focus group & 1 & $0,81 \%$ \\
Outro & 14 & $11,29 \%$ \\
\hline
\end{tabular}

Em relação ao modo de coleta de dados aplicado nas pesquisas survey (vide Tabela 7), observou-se que o questionário aplicado foi a estratégia mais utilizada $(39,52 \%)$, seguido do questionário enviado pelo correio $(27,42 \%)$. Salientase que em 4 artigos analisados não foi possível identificar como os dados foram coletados.

Tabela 7: Tipo de Instrumento, Estratégia de Aplicação e Modo de Coleta - Etapa Quantitativa

\begin{tabular}{lcc}
\hline Instrumento & $\mathbf{N}^{(2)}$ & Perc. \\
\hline Ausente & 4 & $3,23 \%$ \\
Questionário aplicado (entrevista) & 49 & $39,52 \%$ \\
Questionário por correio & 34 & $27,42 \%$ \\
Questionário entregue/recolhido & 14 & $11,29 \%$ \\
Pesquisa documentacional & 5 & $4,03 \%$ \\
Questionário eletrônico & 4 & $3,23 \%$ \\
Questionário por telefone & 2 & $1,61 \%$ \\
Pesquisa bibliográfica & 2 & $1,61 \%$ \\
Outro & 17 & $13,71 \%$ \\
\hline
\end{tabular}


A Tabela 8 demonstra que os trabalhos analisados, na sua maioria, utilizaram dados colhidos especificamente para a pesquisa descrita, primários ou ambos $(93,28 \%)$, sendo que apenas $6,72 \%$ das pesquisas se basearam exclusivamente em dados secundários.

\section{Tabela 8: Freqüência de Artigos por Tipo de Dado Coletado}

\begin{tabular}{lcc}
\hline Tipo de dados & N & Perc. \\
\hline Primários & 106 & $79,10 \%$ \\
Secundários & 9 & $6,72 \%$ \\
Ambos & 19 & $14,18 \%$ \\
\hline Total & $\mathbf{1 2 4}$ & \\
\hline
\end{tabular}

Em relação ao processo de amostragem (vide Tabelas 9 a 11), conclui-se que a utilização de amostras é geralmente o caminho escolhido pelas pesquisas survey, uma vez que em apenas $4,84 \%$ dos artigos se utilizou o censo. No que diz respeito ao tipo de amostra utilizado, observa-se um predomínio das amostras não probabilísticas (81,90\% das amostragens), principalmente das amostras de retorno de correio $(29,03 \%)$ e de conveniência (20,16\%). Verifica-se, ainda, que em 53,23\% dos artigos analisados não foi apresentada a caracterização da amostra do estudo.

No que tange ao teste do viés dos não respondentes (vide Tabela 12), observase que este teste foi realizado em apenas 5,65\% dos artigos analisados $(9,46 \%$ dos casos em que o teste seria aplicável), demonstrando pouca preocupação em avaliar se os não respondentes diferiam significativamente daqueles que participaram da pesquisa.

Tabela 9: Freqüência de Censos e Amostras

\begin{tabular}{lcc}
\hline Censo x Amostra & N & Perc. \\
\hline Ausente & 13 & $10,48 \%$ \\
Censo & 6 & $4,84 \%$ \\
Amostra & 105 & $84,68 \%$ \\
\hline Total & $\mathbf{1 2 4}$ & \\
\hline
\end{tabular}


Tabela 10: Freqüência por Tipo de Amostragem

\begin{tabular}{|c|c|c|c|c|c|}
\hline T ipo de A m ostra & $\mathrm{N}$ & Perc. & $M$ étodo & $\mathrm{N}$ & Perc. \\
\hline A u sente & 9 & $8,57 \%$ & & & \\
\hline \multirow[t]{6}{*}{ Probabilística } & \multirow[t]{6}{*}{10} & \multirow[t]{6}{*}{$9,52 \%$} & A leatórí simples & 2 & $20,00 \%$ \\
\hline & & & A kató ria estratificad a & 4 & $40,00 \%$ \\
\hline & & & A kató ria em agrupam ento $\mathrm{s}$ & 1 & $10,00 \%$ \\
\hline & & & A bató ria sistem ática & 1 & $10,00 \%$ \\
\hline & & & A lató ria por área & 1 & $10,00 \%$ \\
\hline & & & o utro & 1 & $10,00 \%$ \\
\hline \multirow[t]{5}{*}{$\mathrm{N}$ ão probabilística } & \multirow[t]{5}{*}{86} & \multirow[t]{5}{*}{$81,90 \%$} & Conveniêncí & 25 & $20,16 \%$ \\
\hline & & & Julgam ento & 8 & $6,45 \%$ \\
\hline & & & Por quaotas & 14 & $11,29 \%$ \\
\hline & & & R eto mo de correi & 36 & $29,03 \%$ \\
\hline & & & O utro & 3 & $2,42 \%$ \\
\hline Total & 105 & & & & \\
\hline
\end{tabular}

Tabela 11: Freqüência de Caracterização da Amostra

\begin{tabular}{lcc}
\hline Caracte rização da Amos tra & N & Perc. \\
\hline Ausente & 66 & $53,23 \%$ \\
Sem comparação com a população & 51 & $41,13 \%$ \\
Com comparação com a população & 1 & $0,81 \%$ \\
Não se aplica & 6 & $4,84 \%$ \\
\hline Total & $\mathbf{1 2 4}$ & \\
\hline
\end{tabular}

Tabela 12: Freqüência de Teste do Viés

\begin{tabular}{lcc}
\hline Teste do Viés & $\mathbf{N}$ & Perc. \\
\hline Sim & 7 & $5,65 \%$ \\
Não & 67 & $54,03 \%$ \\
Não se aplica & 50 & $40,32 \%$ \\
\hline Total & $\mathbf{1 2 4}$ & \\
\hline
\end{tabular}

\section{Medida da Confiabilidade e Validade dos Constructos}

As dimensões englobadas nesta categoria incluem a confiabilidade e a validade 
dos constructos (validade convergente e discriminante). As Tabelas 13 e 14 descrevem os resultados dessa categoria.

\section{Tabela 13: Freqüência de Tipo de Análise para Teste da Confiabilidade}

\begin{tabular}{lcc}
\hline Confiabilidade & N & Perc. \\
\hline Ausente & 94 & $75,81 \%$ \\
Alpha de Cronbach & 22 & $17,74 \%$ \\
Outro & 8 & $6,45 \%$ \\
\hline Total & $\mathbf{1 2 4}$ & \\
\hline
\end{tabular}

Em relação à confiabilidade, que consiste em determinar para o instrumento de coleta de dados o quanto da variação dos escores é devido às inconsistências na medição (Churchill Jr., 1999), permitindo correlacionar os resultados de uma medida com sua própria reprodução, levou-se em consideração se o artigo apresentava ou não o teste, verificando, ainda, que teste de confiabilidade foi utilizado.

\section{Tabela 14: Freqüência de Validade Convergente e Discriminante}

\begin{tabular}{llcc}
\hline & & N & Perc. \\
\hline Validade Convergente & Sim & 10 & $8,06 \%$ \\
\cline { 2 - 4 } & Não & 114 & $91,94 \%$ \\
\hline Validade Discriminante & Sim & 7 & $5,65 \%$ \\
\cline { 2 - 4 } & Não & 117 & $94,35 \%$ \\
\hline
\end{tabular}

A validade de constructo visa a definir quais as dimensões que estão sendo mensuradas (Malhotra, 1996) e o que de fato o instrumento está medindo (Churchill Jr., 1999), podendo ser verificada, de acordo com Hair Jr. et al. (1998), quando a correlação entre duas medidas do mesmo constructo, realizada com métodos diferentes, é elevada e significativa (validade convergente) e quando a correlação entre indicadores de diferentes constructos é baixa e não significativa (validade discriminante). Considerou-se, para avaliar a validade de constructo, se o artigo apresentava ou não validade convergente e validade discriminante.

As Tabelas 13 e 14 permitem concluir que, de maneira geral, existe pouca preocupação em verificar (ou pelo menos descrever) a confiabilidade e validade dos constructos. Apenas uma pequena parte dos artigos analisados apresentou avaliação da confiabilidade $(24,19 \%)$ e das validades convergente $(8,06 \%)$ e discriminante $(5,65 \%)$. 


\section{Tipos de Análise de Dados}

Apresenta-se aqui a freqüência de cada tipo de análise de dados utilizado nas pesquisas survey. A Tabela 15 resume os resultados da análise dos artigos.

Tabela 15: Freqüência de Tipos de Análise de Dados

\begin{tabular}{lcc}
\hline Tipo de Análise & $\mathbf{N}^{(2)}$ & Perc. \\
\hline Freqüência & 59 & $47,58 \%$ \\
Média & 49 & $39,52 \%$ \\
Fatorial & 31 & $25,00 \%$ \\
Anova/testet & 27 & $21,77 \%$ \\
Regressão (correlação) & 26 & $20,97 \%$ \\
Desvio padrão & 21 & $16,94 \%$ \\
Qui-quadrado & 20 & $16,13 \%$ \\
Discriminante & 11 & $8,87 \%$ \\
Mediana & 10 & $8,06 \%$ \\
Regressão (múltipla) & 7 & $5,65 \%$ \\
Moda & 2 & $1,61 \%$ \\
Outro & 47 & $37,90 \%$ \\
\hline
\end{tabular}

A constatação neste item é a de que os tipos de análises de dados utilizados nos artigos analisados foram preferencialmente as estatísticas descritivas como freqüência (utilizada em 47,58\% dos estudos) e média (utilizada em 39,52\% dos estudos).

\section{Forma de Apresentaçăo dos Resultados}

Relata-se aqui a forma com que os resultados de pesquisa foram apresentados nos artigos analisados, isto é, se eles indicaram a validade externa e nomológica, bem como os limites do estudo e as recomendações aplicadas e para futuras pesquisas. A Tabela 16 apresenta os resultados dessa categoria.

Quanto à validade externa, buscou-se avaliar se os artigos descreviam em que medida os resultados obtidos poderiam ser aplicados a outras situações, além daquelas referentes ao estudo apresentado (Churchill Jr., 1999).

Já no item relativo à validade nomológica, considerou-se a presença ou não de uma descrição da consistência entre resultados obtidos e as hipóteses definidas $a$ priori, a teoria correspondente e resultados de pesquisas anteriores (Sampieri, Collado e Lucio, 1998; Churchill Jr., 1999). 
Em relação aos limites do estudo e recomendações para pesquisas, verificouse apenas a sua presença ou não nos artigos.

$\mathrm{Na}$ Tabela 16, verifica-se que a preocupação dos autores com validade externa e nomológica pode ser considerada pequena. Apenas 14,52\% dos artigos descreveram aspectos relacionados à validade externa e $16,94 \%$ relacionados à validade nomológica.

\section{Tabela 16: Freqüência de Artigos por Tipo de Dado Coletado}

\begin{tabular}{llcc}
\hline Validade externa & & N & Perc. \\
\hline Validade nomológica & Sim & 18 & $14,52 \%$ \\
\cline { 2 - 4 } & Não & 106 & $85,48 \%$ \\
\hline Limites do estudo & Sim & 21 & $16,94 \%$ \\
\cline { 2 - 4 } & Não & 103 & $83,06 \%$ \\
\hline Recomendações para pesquisas futuras & Sim & 47 & $37,90 \%$ \\
\cline { 2 - 4 } & São & 77 & $62,10 \%$ \\
\cline { 2 - 4 } & Não & 71 & $53,74 \%$ \\
\hline Recome ndações aplicadas & Sim & 94 & $75,81 \%$ \\
\cline { 2 - 4 } & Não & 30 & $24,19 \%$ \\
\hline
\end{tabular}

Nota-se, ainda, que os autores dão maior ênfase às recomendações aplicadas $(75,81 \%$ dos artigos) do que às recomendações de pesquisas futuras $(42,74 \%$ dos artigos). Isto pode denotar uma prioridade para resultados aplicáveis diretamente na prática do marketing em detrimento de resultados teórico-acadêmicos. O volume de artigos que não descreve as limitações do estudo $(62,10 \%)$ parece corroborar esta conclusão.

\section{Cruzamento de Variáveis}

$\mathrm{Na}$ análise dos dados colhidos, os resultados de algumas variáveis foram cruzados, objetivando um aprofundamento no diagnóstico da situação das publicações brasileiras na área de marketing.

Inicialmente, considerou-se a natureza de pesquisa e suas relações com outras variáveis. Quanto à natureza exploratória, considera-se que esta é apropriada para qualquer problema ou fenômeno sobre o qual pouco se sabe, servindo como fundamentação para futuros estudos (Sampieri, Collado e Lucio, 1998; Churchill Jr., 1999). Esta ponderação não se evidencia no levantamento feito. Dos 37 arti- 
gos de natureza exploratória, apenas $16(43,24 \%)$ manifestaram expressamente recomendações para pesquisas futuras.

Já o estudo descritivo pressupõe um conhecimento anterior do fenômeno estudado, apoiando-se em uma ou mais hipóteses que guiam a pesquisa em direções específicas (Sampieri, Collado e Lucio, 1998; Churchill Jr., 1999). Também neste caso os dados não condizem com o pressuposto inicial. Dos 76 artigos de natureza descritiva, apenas $25(32,89 \%)$ apresentaram hipóteses e pressupostos de base, 9 $(11,84 \%)$ não discutiram as teorias de base, $40(52,63 \%)$ as discutiram superficialmente e apenas $27(52,63 \%)$ com profundidade.

A pesquisa de natureza causal, por sua vez, vai além da descrição de fenômenos ou do estabelecimento de relações entre conceitos, exigindo uma estruturação anterior maior que as outras naturezas de pesquisa (Sampieri, Collado e Lucio, 1998; Churchill Jr., 1999). O levantamento apontou 11 artigos de natureza causal, dos quais 9 apresentaram hipóteses formuladas, 7 discutiram superficialmente as teorias de base e apenas $4(36,36 \%)$ de forma aprofundada. Estes números se aproximam da idéia de qualidade em artigos de natureza causal. Contudo, outras variáveis demonstram uma realidade não tão favorável. O estudo registrou que 3 dos 11 artigos de natureza causal não apresentaram um modelo teórico de pesquisa e, ainda, 8 não registraram a avaliação de validade convergente, 9 não demonstraram a validade discriminante e 7 não fizeram referência à validade nomológica.

No que diz respeito à validade preditiva das pesquisa apresentadas, os dados demonstram reduzida atenção ao tipo de amostra utilizado. Dos 11 artigos de natureza causal, 10 registraram pesquisas com amostras não-probabilísticas: 4 retornos de correio, 3 por julgamento, 2 por conveniência e 1 por cotas. Quanto à natureza descritiva, dos 76 artigos identificados nesta natureza, apenas 58 (76,31\%) registraram o tipo de amostra utilizado, sendo que destes 51 (87,93\%) declaram o uso de amostras não-probabilísticas. Nestes casos, a concentração de tipos de amostra ficou em retorno de correio (21), conveniência (17) e por cotas (8).

Em função do elevado número de aplicações do meio de coleta quantitativo questionário enviado por correio (presente em cerca de 27\% dos 124 artigos), avaliou-se a relação desta variável com a realização do teste de viés dos respondentes (Churchill Jr., 1999). Dos 34 artigos que descreveram a utilização de questionários enviados por correio, somente 7 apresentaram algum tipo de resultado de teste de viés.

Mais especificamente sobre o instrumento de coleta - questionário, entendese que este instrumento deve ser elaborado por uma devida fase exploratória, seguido de uma validação de conteúdo e de sua respectiva análise de confiabi- 
lidade (Malhotra, 1996; Churchill Jr., 1999). A Tabela 17 demonstra a relativa fragilidade dos instrumentos apresentados pelos artigos analisados. Em geral, apesar de boa parte dos questionários ter passado por uma etapa exploratória (declarada) de preparação, menos da metade teve a validade de conteúdo avaliada e um número bastante reduzido deles apresentou confiabilidade testada.

\section{Tabela 17: Freqüência de Fase Exploratória, Validade de Conteúdo e Confiabilidade para o Método de Coleta - Questinários}

\begin{tabular}{lcccc}
\hline & Total & $\begin{array}{c}\text { Fase } \\
\text { Exploratória }\end{array}$ & $\begin{array}{c}\text { Validade de } \\
\text { Conte údo }\end{array}$ & Confiabilidade \\
\hline Questionário por correio & 34 & $20(58,8 \%)$ & $16(47,1 \%)$ & $9(26,5 \%)$ \\
\hline Questionário aplicado & 49 & $29(59,2 \%)$ & $23(46,9 \%)$ & $16(32,6 \%)$ \\
\hline Questionário entregue/re colhido & 14 & $11(78,6 \%)$ & $6(42,85 \%)$ & $2(14,3 \%)$ \\
\hline Questionário tele fone & 2 & $1(50,0 \%)$ & $0(0 \%)$ & $0(0 \%)$ \\
\hline Questionário ele trônico & 4 & $4(100 \%)$ & $2(50,0 \%)$ & $2(50,0 \%)$ \\
\hline
\end{tabular}

\section{CONCLUSÓES}

A análise dos artigos de pesquisas survey publicados na RAE, na RAUSP e no ENANPAD, no período de janeiro de 1990 a dezembro de 1999, em termos gerais, sinaliza pouca preocupação com aspectos relevantes da qualidade metodológica da investigação. Ficou evidente a baixa consideração aos seguintes elementos metodológicos: hipóteses e pressupostos básicos, modelo de pesquisa, mix de método, operacionalização das variáveis, validade de conteúdo, amostragem probabilística, teste do viés de não-respondentes, confiabilidade, validade convergente, validade discriminante, validade externa e nomológica, limites do estudo e recomendações para pesquisas futuras.

Esta constatação, em princípio, poderia ser justificada pelo limite de páginas imposto pelo principal periódico analisado nesta pesquisa (15 páginas), pela inabilidade de síntese dos autores ou pela orientação para resultados práticos, mais distantes do rigor metodológico.

Salienta-se que o percentual de pesquisas tipo survey de natureza causal $(8,87 \%)$ ainda é muito pequeno em relação às pesquisas de natureza exploratória $(29,84 \%$ dos artigos) e descritiva (61,29\% dos artigos). Apenas 11 artigos (dos 124 artigos analisados) objetivaram testar teorias e suas relações causais. A busca pela consolidação da área de marketing no Brasil e a correspondente necessidade de 
conhecimento de suas peculiaridades expressa-se, claramente, pela concentração de pesquisas de natureza descritiva.

Nesta linha, poder-se-ia argumentar que, na medida que a intensidade dos estudos se voltar para a natureza causal, o rigor metodológico e a qualidade das pesquisas tipo survey deva crescer.

\section{Limites do Estudo}

Pode-se relacionar como limites deste estudo os seguintes pontos.

- As conclusões do estudo estão restritas aos três periódicos (RAE, RAUSP e ENANPAD) e ao período (01/1990 a 12/1999) considerados.

- A subjetividade implícita de cada avaliador em suas análises individuais pode ter representado conclusões distintas para uma mesma situação, apesar dos critérios claros de análise e das reuniões de revisão de dúvidas.

- Os resultados aqui descritos consideraram simultânea e concomitantemente artigos produzidos em momentos distintos no tempo, desconsiderando, portanto, uma eventual evolução longitudinal da produção científica analisada.

\section{Pesquisas Futuras}

A partir dos resultados deste estudo, sugerem-se as seguintes pesquisas futuras.

. Comparação dos resultados da área de marketing com outras áreas.

. Comparação dos resultados nacionais com resultados de outros países.

- Avaliação da evolução longitudinal da qualidade da pesquisa científica no conjunto de artigos analisados.

\section{NotAs}

${ }^{1}$ Em geral, Bertero, Caldas e Wood Jr. (1998 - Organizações), Carrieri e Luz (1998 - Organizações), Hoppen et al. (1998 - Administração da Informação) e Vieira (1998, 1999 - Marketing), além da organização de painéis e mesas redondas nas áreas de Organizações, Marketing e Recursos Humanos.

$2 \mathrm{O}$ total de casos ultrapassa o número de 124 artigos, uma vez que alguns artigos utilizaram mais de um método ou instrumento. 


\section{Anexo 1: Survey}

\begin{tabular}{|c|c|c|c|c|}
\hline \multicolumn{5}{|l|}{ CLASSIFICAÇÃO: } \\
\hline \multicolumn{5}{|l|}{ AUTOR (ES) : } \\
\hline \multicolumn{5}{|l|}{$\begin{array}{l}\text { TÍTULO : } \\
\text { PERIÓDICO }\end{array}$} \\
\hline \multicolumn{5}{|l|}{ PERIÓDICO : } \\
\hline NATUREZA & Natureza da pesquisa & \multicolumn{3}{|c|}{\lrcorner Exploratória $\quad \sqcup$ Descritiva $\quad \sqcup$ Causal } \\
\hline \multirow{4}{*}{$\begin{array}{l}\text { QUEST ÃO } \\
\text { DE PESQUISA }\end{array}$} & Justificativa & \multicolumn{3}{|c|}{$\sqsupset \operatorname{Sim} \quad \square$ Não } \\
\hline & Teorias de base & \multicolumn{3}{|c|}{$\begin{array}{l}\sqsupset \text { Aprofundada } \quad \square \text { Superficial } \\
\lrcorner \text { Ausente ou não relacionada }\end{array}$} \\
\hline & Objetivo/Questão de pesquisa & \multicolumn{3}{|c|}{\begin{tabular}{|l|} 
Ausente ou nao relacionada \\
Sim
\end{tabular}} \\
\hline & Hipóteses / pressupostos de base & $\sqsupset \operatorname{Sim}$ & \multicolumn{2}{|l|}{$\square$ Não } \\
\hline \multirow{7}{*}{$\begin{array}{l}\text { DESENHO } \\
\text { DE PESQUISA }\end{array}$} & Modelo de pesquisa & \lrcorner $\operatorname{Sim}$ & \multicolumn{2}{|l|}{ பNão } \\
\hline & \begin{tabular}{|l|} 
Típo de estudo \\
\end{tabular} & \lrcorner Longitudinal & \multicolumn{2}{|c|}{$\sqcup$ Corte Transversal } \\
\hline & Mix de métodos & $\sqsupset \operatorname{Sim}$ & \multicolumn{2}{|c|}{ QNão } \\
\hline & Objeto da investigação (Qual) & \multicolumn{3}{|c|}{$x^{2}+2$} \\
\hline & Sujeito da investigação (Qual) & & \multirow{2}{*}{\multicolumn{2}{|c|}{$\square$ Não }} \\
\hline & Operacionalização das variáveis & $\sqsupset \mathrm{Sim}$ & & \\
\hline & Descrição dos procedimentos metodológicos & $\sqsupset \mathrm{Sim}$ & \multicolumn{2}{|l|}{$\square$ Não } \\
\hline \multirow[t]{10}{*}{$\begin{array}{l}\text { INSTRUMENTOS DE } \\
\text { MEDIDA E COLETA DOS } \\
\text { DADOS }\end{array}$} & $\begin{array}{l}\text { Tipo de instrumento, estratégia de aplicação e } \\
\text { modo de coleta - Etapa Exploratória }\end{array}$ & $\begin{array}{l}\lrcorner \text { Ausente } \\
\sqsupset \text { Focus group } \\
\sqsupset \text { Outro; }\end{array}$ & \multicolumn{2}{|c|}{$\begin{array}{l}\sqcup \text { Entrevista em profundidade } \\
\square \text { Instr. gerado em outra pesq. }\end{array}$} \\
\hline & Validade do conteúdo / face & \begin{tabular}{|l}
$\neg$ Ausente \\
$\neg$ Pré-teste \\
\lrcorner Não se aplica \\
\end{tabular} & \multicolumn{2}{|l|}{$\begin{array}{l}\sqcap \text { Focus group } \\
\sqcup \text { Outro: }\end{array}$} \\
\hline & $\begin{array}{l}\text { Tipo de instrumento, estratégia de aplicação e } \\
\text { modo de coleta - Etapa Quantitativa }\end{array}$ & \multicolumn{3}{|c|}{$\begin{array}{l}\sqsupset \text { Questionário por correio } \\
\sqsupset \text { Questionário aplicado (entrevista) } \\
\sqsupset \text { Questionário entregue / recolhido } \\
\sqsupset \text { Questionário por telefone } \\
\sqsupset \text { Questionário eletrônico } \\
\sqsupset \text { Pesquisa documentacional } \\
\neg \text { Pesquisa bibliográfica } \\
\sqsupset \text { Outro: }\end{array}$} \\
\hline & \begin{tabular}{|l|} 
Tipo de dados \\
\end{tabular} & \multirow{2}{*}{\multicolumn{3}{|c|}{$\sqsupset$ Primários $\quad \square$ Secundários }} \\
\hline & Respondentes (Quais) & & & \\
\hline & Tamanho da amostra (Número) & \\
\hline & Tipo de amostra & Não probabilistica: & \multicolumn{2}{|c|}{$\begin{array}{l}\square \text { Aleatória Simples } \\
\square \text { Aleatória Estratificada } \\
\sqcap \text { Aleatória em Agrupamentos } \\
\square \text { Aleatória Sistemática } \\
\square \text { Aleatória por Área } \\
\sqcap \text { Outro; } \\
\square \text { Conveniência } \\
\square \text { Julgamento } \\
\sqcup \text { Por Quotas } \\
\square \text { Retorno de correio } \\
\square \text { Outro: }\end{array}$} \\
\hline & Taxa de resposta (\%) & $\square$ Ause & \multicolumn{2}{|c|}{ Ausente $\quad \square$ Não se aplica } \\
\hline & 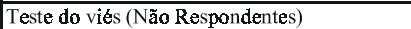 & $\square$ Não & o $\quad \sqsubset$ Não se & e aplica \\
\hline & Caracterização da amostra & $\begin{array}{l}\sqsupset \text { Sem comparação } \\
\lrcorner \text { Com comparação }\end{array}$ & $\begin{array}{l}\text { o com a população } \\
\text { o com a população }\end{array}$ & $\begin{array}{ll}0 \\
0\end{array}$ \\
\hline VALIDADE DOS & Confiabilidade & $\sqsupset$ Ausente $\quad \square \mathrm{Al}$ & Alpha de Cronbach & $\square$ Outro: \\
\hline CONSTRUTOS & Validade convergente & $\sqsupset \mathrm{Sim}$ & पNão & \\
\hline & Validade discriminante & \lrcorner $\operatorname{Sim}$ & பNão & \\
\hline ANÁLISE DOS DADOS & Tipos de análise (Quais) & \begin{tabular}{|ll}
$\neg$ Freqüência & $\neg \mathrm{\Lambda}$ \\
\lrcorner Mediana & \lrcorner $\mathrm{I}$ \\
$\neg$ Fatorial & $\sqsupset$ \\
\lrcorner Regres. Mult. & \lrcorner $\mathrm{I}$ \\
\end{tabular} & $\begin{array}{l}7 \text { Média } \\
\text { Desvio padrão } \\
\text { Anova / Teste t } \\
\text { J Discriminante }\end{array}$ & $\begin{array}{l}\sqcap \text { Moda } \\
\sqcup \text { Correlação } \\
\square \text { Qui-quadrado } \\
\sqcup \text { Outro: }\end{array}$ \\
\hline APRESENT AÇÃO & \begin{tabular}{|l|} 
Validade externa \\
\end{tabular} & $\sqsupset \operatorname{Sim}$ & $\square$ Não & \\
\hline DOS RESULTADOS & Validade nomológica & $\sqsupset \operatorname{Sim}$ & $\square$ Não & \\
\hline & \begin{tabular}{|l|} 
Limites do estudo \\
\end{tabular} & $\sqsupset \operatorname{Sim}$ & प Nào & \\
\hline & Recomendaçóes para pesquisas & $\sqsupset \operatorname{Sim}$ & $\square$ Não & \\
\hline & \begin{tabular}{|l|} 
Recomendações aplicadas \\
\end{tabular} & $\sqsupset \operatorname{Sim}$ & $\square$ Não & \\
\hline
\end{tabular}




\section{ReferênCias Bibliográficas}

CHURCHILL JR., G. A. Marketing research : methodological foundation. Orlando, FL : The Dryden Press, 1999.

HAIR JR., J. et al.

Multivariate data analysis. Englewood Cliffs, NJ : PrenticeHall, 1998.

HOPPEN, N. et al.

Avaliação de artigos de pesquisa em sistemas de informação : proposta de um guia. In: XXI
ENCONTRO ANUAL DA ANPAD (1997 : Angra dos Reis). Anais Eletrônicos... Rio de Janeiro : ANPAD, 1997.

MALHOTRA, N. K.

Marketing research : an applied orientation. Englewood Cliffs, NJ : Prentice-Hall, 1996.

SAMPIERI, R. H.;

COLLADO, C. F.;

LUCIO, P. B.

Metodología de la investigación. México : McGraw-Hill, 1998. 